\title{
Two-Port Transmission Line Technique for Dielectric Property Characterization of Polymer Electrolyte Membranes
}

\author{
Zijie Lu, ${ }^{\dagger, l l}$ Michael Lanagan, ${ }^{\ddagger}$ Evangelos Manias, ${ }^{*, \S}$ and Digby D. Macdonald ${ }^{*, \dagger}$ \\ Center for Electrochemical Science and Technology, Department of Materials Science and Engineering, Center \\ for Dielectric Studies, Materials Research Lab, and Polymer Nanostructures Lab, Department of Materials \\ Science and Engineering, Penn State University, University Park, Pennsylvania 16802
}

Received: June 17, 2009; Revised Manuscript Received: August 26, 2009

\begin{abstract}
Performance improvements of perfluorosulfonic acid membranes, such as Nafion and Flemion, underline a need for dielectric characterization of these materials toward a quantitative understanding of the dynamics of water molecules and protons within the membranes. In this Article, a two-port transmission line technique for measuring the complex permittivity spectra of polymeric electrolytes in the microwave region is described, and the algorithms for permittivity determination are presented. The technique is experimentally validated with liquid water and polytertrafluoroethylene film, whose dielectric properties are well-known. Further, the permittivity spectra of dry and hydrated Flemion SH150 membranes are measured and compared to those of Nafion 117. Two water relaxation modes are observed in the microwave region $(0.045-26 \mathrm{GHz})$ at $25{ }^{\circ} \mathrm{C}$. The higher-frequency process observed is identified as the cooperative relaxation of bulk-like water, whose amount was found to increase linearly with water content in the polymer. The lower-frequency process, characterized by longer relaxation times in the range of $20-70 \mathrm{ps}$, is attributed to water molecules that are loosely bound to sulfonate groups. The loosely bound water amount was found to increase with hydration level at low water content and levels off at higher water contents. Flemion SH150, which has an equivalent weight of 909 g/equiv, displays higher dielectric strengths for both of these water modes as compared to Nafion 117 (equivalent weight of 1100 g/equiv), which probably reflects the effect of equivalent weight on the polymers' hydrated structure, and in particular its effect on the extended ionic cluster domains.
\end{abstract}

\section{Introduction}

Perfluorosulfonic acid (PFSA) polymer electrolytes have attracted much attention over the past decade because of their applications in polymer electrolyte membrane fuel cells (PEMFC), as well as in other electrochemical devices such as electrochemical sensors and water electrolyzers. ${ }^{1-3}$ Especially, their application to PEMFC has led to extensive research of their structure-property relations. Nafion (a DuPont registered trademark polymer) has become the prototypical polymer electrolyte because it combines high proton conductivity with a high electrochemical stability and good thermomechanical properties. Nafion combines, in one molecule, a hydrophobic backbone and hydrophilic sulfonic-acid functionalized side groups, which promote a microphase separation between the polar (hydrophilic) and nonpolar (hydrophobic) constituents. ${ }^{4,5}$ In its hydrated form, Nafion's hydrophilic (nano)phases contain water and acidic groups and can become interconnected to provide pathways for the transport of dissociated protons; concurrently, the hydrophobic domains, consisting of the fluorocarbon backbones, provide good mechanical and thermal stability. The structure and morphology of Nafion have been reported in several comprehensive reviews. ${ }^{6,7}$

PFSA membranes are essentially water-based electrolytes because they are only operational when sufficient amount of

* Corresponding author. D.D.M.: Tel., (814) 863-7772; fax, (814) 8634718; e-mail,ddm2@psu.edu. E.M.: E-mail, manias@ psu.edu.

† Center for Electrochemical Science and Technology.

* Center for Dielectric Studies.

$\S$ Polymer Nanostructures Lab.

"Current address: Department of Mechanical Engineering, Rochester Institute of Technology, Rochester, NY 14623. water is absorbed within the polymer and around the sulfonicacid groups. This particular feature enables the use of dielectric relaxation spectroscopy (DRS), which monitors the cooperative motion of polar molecular assemblies, providing a powerful tool for the characterization of these materials. DRS probes the response of the total dipole moment of a system, $M(t)=\sum \mu_{i}(t)$, inside a time-dependent external electrical field; $\mu_{i}$ represents the individual molecular dipole moments. The dielectric properties of a material are described by the complex relative permittivity spectrum:

$$
\varepsilon^{*}(\omega)=\varepsilon^{\prime}(\omega)-j \varepsilon^{\prime \prime}(\omega)
$$

with $\varepsilon^{\prime}(\omega)$ denoting the relative permittivity, and $\varepsilon^{\prime \prime}(\omega)$ the dielectric loss (or absorption), both being functions of frequency $(\omega=2 \pi f) ; j=\sqrt{ }-1$ is the imaginary unit. The dielectric properties of polymer electrolytes are dictated by the molecular structure of the system and the physical state of water in these materials. ${ }^{8-11}$ As such, dielectric spectra can provide insights into both the state of water and, indirectly, the hydrated structure of the polymer. Furthermore, the dielectric relaxation behavior provides correlations between the polymer environment and the cooperative solvent dynamics, which can unveil important insights on the water and proton transport in these membranes. ${ }^{12-14}$ It is widely accepted that water confined in systems such as reverse micelles possesses different dynamics as compared to bulk/free water. As structural diffusion (i.e., the Grotthuss mechanism) of protons in bulk water requires coordinated formation and cleavage of hydrogen bonds of water molecules in the second hydration shell of the hydrated proton (Zundel 
and Eigen ions ${ }^{15,16}$ ) and, thus, requires rotational relaxation, consequently, any constraint to the dynamics of the water molecules will decrease the mobility of the protons.

However, despite their obvious importance, the available dielectric data for PFSA polymer electrolytes are rather limited. Yeo and Eisenberg ${ }^{17}$ carried out the dynamic mechanical and dielectric studies of hydrated perfluorosulfonate polymers and observed dielectric dispersions in the $\beta$ relaxation region, which were postulated to be related to the polar regions. Similar relaxation process was also observed in $\mathrm{K}^{+}$-exchanged Nafion ${ }^{18,19}$ and in Nafion $/\left(\mathrm{M}_{x} \mathrm{O}_{y}\right)_{n}$ composite membrane. ${ }^{20}$ Mauritz and co-workers ${ }^{21,22}$ reported constant temperature dielectric relaxation analyses of Nafion membranes imbibed with various ions attempting to model long-range ion transport. These investigations were limited to low frequencies $(f<10 \mathrm{MHz})$, and no specific information about the water dynamics was observed. Starkweather and Chang ${ }^{23}$ observed water dielectric relaxation in the low-frequency range at low temperatures. However, for aqueous and water-based electrolytes at ambient temperature, the dielectric relaxation of water-containing regions is in the microwave frequency region, and its measurement requires special instrumentation. The high specific electric conductivity, $\sigma$, of these systems presents another challenge because only the total loss of the sample

$$
\eta^{\prime \prime}(\omega)=\varepsilon^{\prime \prime}(\omega)+\eta_{\sigma}^{\prime \prime}=\varepsilon^{\prime \prime}(\omega)+\frac{\sigma}{\omega \varepsilon_{0}}
$$

is experimentally accessible; $\varepsilon_{0}$ is the permittivity of the vacuum. Obviously, the ohmic loss, $\eta_{\sigma}^{\prime \prime}$, dominates the total dielectric loss below a minimum frequency, which is characteristic of each system, and in the case of hydrated PFSA usually lies in the radio frequency $(\mathrm{RF})$ and microwave frequency ranges, thus limiting the spectral range accessible to experiments. ${ }^{11}$ Therefore, there is a clear need for accurate high-frequency (microwave) dielectric characterization techniques, capable of robust quantitative measurement of the dielectric properties of various electrolytes, including aqueous and polymeric materials.

Currently, there are several available microwave dielectric property measurement techniques. The open-ended coaxial probe technique enables convenient and nondestructive measurements of the dielectric properties of infinite half-space solids and liquids ${ }^{24-26}$ and has been extended to the measurements of finitethickness layered materials. ${ }^{24,27}$ Paddison et al. ${ }^{28,29}$ used the technique to measure the dielectric properties of Nafion 117 in the frequency range of $0.045-30 \mathrm{GHz}$. However, the employment of a reflection geometry yielded very poor quality permittivity spectra, and, thus, no detailed information about discrete water relaxations could be obtained, beyond the expected dependence of the dielectric constant and of the loss factors on the water content. ${ }^{28,29}$ Moreover, improper contact between the probe aperture and the material surface can result in significant errors in measuring its dielectric properties. Resonant cavity methods render accurate measurements, but they are not broadband and are generally limited to low loss values $(\tan \delta<0.02)$. On the other hand, transmission line techniques are relatively accurate in measuring the broadband dielectric properties of intermediate and high loss dielectric materials. Of these, the two-port transmission line techniques ${ }^{30,31}$ are presently widely used. In general, these techniques make use of the reflected and/or transmitted waves by and through a dielectricfilled transmission line to analytically or numerically determine the dielectric properties of the material. In addition, the twoport transmission line techniques are well suited for the dielectric measurement of polymeric electrolytes. In this case, a precisely cut sample is placed in a section of a transmission line, and the complex scattering parameters are measured over a wide frequency range. The scattering equations relate the reflected/ transmitted waves to the permittivity and permeability of the material. The dielectric properties of a material are then determined analytically or numerically from the relevant scattering parameters.

In our previous work, we have studied the dielectric relaxation spectroscopy of Nafion 117 combining normal broadband dielectric spectroscopy (for low frequencies) and two-port microwave transmission line method (for microwave frequencies). ${ }^{11}$ In that work, we directly observed and identified at least two water relaxation mechanisms in the microwave frequency range within the hydrated membrane, in addition to a third, much slower, mode from the strongly bound water in the hydration shells around the acidic groups. ${ }^{11}$ In this present work, the emphasis is on further development of this microwave frequency transmission technique, including examining the scattering equations in detail, presenting a simplified iterative numerical method for obtaining the dielectric spectra, and validating the technique using pure water and PTFE film whose dielectric properties are well-known. A second goal of this Article is to comparatively investigate the dielectric relaxation behavior of an additional PFSA membrane having different equivalent weight (EW) from Nafion-11x. It is believed that EW is an important factor in determining the structure of the membrane and the dynamics of protons and water molecules within the membrane. For this purpose, the dielectric spectra of Flemion $\mathrm{SH} 150$, which has a chemical structure similar to that of Nafion 117 but has a lower equivalent weight $(\mathrm{EW}=909$ g/equiv vs EW $=1100$ for Nafion-11x), were measured using the two-port transmission line technique and compared to those of Nafion 117.

\section{Theoretical Background}

2.1. Theory. The main part of the experimental setup is shown in Figure 1a. In this setup, a dielectric sample is installed in a coaxial air-filled line with characteristic impedance $Z_{0}$. Within the sample region, the line will assume a new characteristic impedance $Z=Z_{0} \sqrt{ }\left(\mu^{*} / \varepsilon^{*}\right)$, where $\varepsilon^{*}$ and $\mu^{*}$ are the complex relative permittivity and permeability of the sample. The reflection coefficient $(\Gamma)$ at the interface between the airfilled line and dielectric-filled line when the material sample is infinite in length is given by

$$
\Gamma=\frac{Z-Z_{0}}{Z+Z_{0}}=\frac{\sqrt{\mu^{*} / \varepsilon^{*}}-1}{\sqrt{\mu^{*} / \varepsilon^{*}}+1}
$$

The dielectric sample can be viewed as a two-port device characterized by the scattering parameters, $S_{11}$ and $S_{21}$, which represent the sum of reflected and transmitted waves, respectively. It is not necessary for the sample to completely fill the full length of the coaxial line. The air-filled waveguide sections (regions I and III in Figure 1a) are included here, to take into account the offset between the calibration plane and the measurement plane. The network analyzer system calibration and measurement are obtained at the reference planes indicated in Figure 1.

The scattering parameters are found from an analysis of the electric field at the sample interfaces. ${ }^{31}$ The fundamental propagation mode for waves in a coaxial system is the transverse 
electromagnetic wave (TEM). The spatial distribution of the electric field in the regions I, II, and III, for an incident field normalized to 1 , can be written as:

$$
\begin{gathered}
E_{\mathrm{I}}(x)=\exp \left(-\gamma_{0} x\right)+C_{1} \exp \left(\gamma_{0} x\right) \\
E_{\mathrm{II}}(x)=C_{2} \exp (-\gamma x)+C_{3} \exp (\gamma x) \\
E_{\mathrm{III}}(x)=C_{4} \exp \left(-\gamma_{0} x\right)
\end{gathered}
$$

where $\gamma$ is the complex propagation constant given by

$$
\gamma=\alpha+j \beta=j \omega \sqrt{\varepsilon^{*} \varepsilon_{0} \mu^{*} \mu_{0}}
$$

where $\varepsilon_{0}$ and $\mu_{0}$ are the permittivity and magnetic permeability of vacuum, and $\omega$ is the angular frequency $(\omega=2 \pi f) . \varepsilon^{*} \varepsilon_{0}$ and $\mu^{*} \mu_{0}$ are the absolute permittivity and magnetic permeability of the medium. In the air-filled regions I and III, $\gamma$ reduces to $\gamma_{0}=j \omega \sqrt{ }\left(\varepsilon_{0} \mu_{0}\right)$. The constants $C_{i}$ are determined by appropriate matching of the tangential electric and magnetic fields at all boundaries:

$$
\begin{aligned}
& \mathrm{E}_{\mathrm{I}}\left(x=L_{1}\right)=\mathrm{E}_{\mathrm{II}}\left(x=L_{1}\right) \\
& \mathrm{E}_{\mathrm{II}}\left(x=L_{1}+L\right)=\mathrm{E}_{\mathrm{III}}\left(x=L_{1}+L\right) \\
& \frac{1}{\mu_{0}} \frac{\partial \mathrm{E}_{\mathrm{I}}}{\partial x}\left(x=L_{1}\right)=\frac{1}{\mu} \frac{\partial \mathrm{E}_{\mathrm{II}}}{\partial x}\left(x=L_{1}\right) \\
& \frac{1}{\mu} \frac{\partial \mathrm{E}_{\mathrm{II}}}{\partial x}\left(x=L_{1}+L\right)=\frac{1}{\mu_{0}} \frac{\partial \mathrm{E}_{\mathrm{III}}}{\partial x}\left(x=L_{1}+L\right)
\end{aligned}
$$

The reflection $\left(S_{11}\right)$ and transmission scattering parameters $\left(S_{21}\right)$ are thus obtained by solving eqs $4-6$ subject to the boundary conditions (eqs 8-11). The explicit expressions are given by

$$
\begin{gathered}
S_{11}=R_{1}^{2}\left[\frac{\Gamma(1-\exp (-2 \gamma L))}{1-\Gamma^{2} \exp (-2 \gamma L)}\right] \\
S_{21}=R_{1} R_{2}\left[\frac{\exp (-\gamma L)\left(1-\Gamma^{2}\right)}{1-\Gamma^{2} \exp (-2 \gamma L)}\right]
\end{gathered}
$$

where $R_{1}$ and $R_{2}$ are the reference plane transformations and are expressed by

$$
\begin{aligned}
& R_{1}=\exp \left(-\gamma_{0} L_{1}\right) \\
& R_{2}=\exp \left(-\gamma_{0} L_{2}\right)
\end{aligned}
$$

It should be noted that the same relations have also been derived through other procedures. ${ }^{32,33}$ In this Article, the dielectric specimens are assumed to be nonmagnetic $\left(\mu^{*}=1\right)$, and, therefore, the reflection coefficient at the interface between the air-filled transmission line and dielectric-filled line can be simplified as:

$$
\Gamma=\frac{1-\sqrt{\varepsilon^{*}}}{1+\sqrt{\varepsilon^{*}}}
$$

2.2. Solving for the Dielectric Properties. The $S$-parameters of the two-port transmission line setup are measured using an HP8510C vector network analyzer. Each $S$-parameter measurement is subsequently corrected for the reference plane transformation (eqs 12-15). The dielectric properties of the specimen are then calculated from these $S$-parameters.

Several algorithms, such as the well-known NWR procedure (Nicolson and Ross ${ }^{32}$ and Weir ${ }^{34}$ ) and an improved transmission/ reflection technique, ${ }^{31}$ have been developed to obtain the permittivity and permeability from scattering measurements. In these procedures, both the reflected and the transmitted $S$ parameters $\left(S_{11}\right.$ and $\left.S_{21}\right)$ are combined, and multiple measurements are needed. However, for high dielectric loss material (such as Nafion or Flemion membranes), measurement of $S_{21}$ allows for a higher accuracy than that of $S_{11} \cdot{ }^{35}$ Therefore, in this work, the dielectric properties are determined by measuring the frequency dependence of the transmission $S$-parameter $S_{21}$.

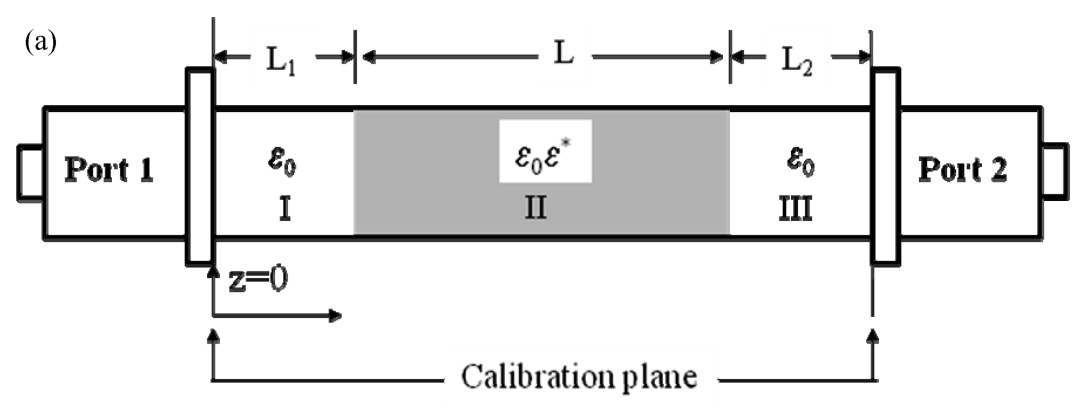

(b)

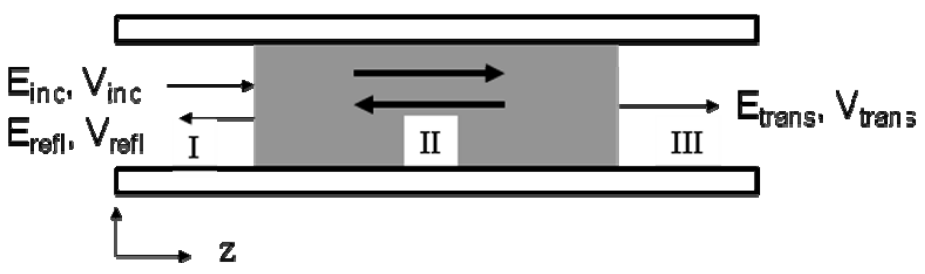

Figure 1. (a) Geometry of the measurement setup for the two-port transmission line technique. Port 1 and port 2 denote calibration reference plane positions. (b) The incidence (inc), reflected (refl), and transmitted (trans) electric field/voltage distributions in regions I, II, and III. 
It should be noted that the analytical solution for the permittivity is difficult to obtain from eq 13, and most often numerical methods are used instead.

$S_{21}$ is usually a complex number and is expressed in terms of magnitude and phase, $S_{21}=\left|S_{21}\right| \mathrm{e}^{j \phi}$. The phase $\phi$ is related to the permittivity $\left(\varepsilon^{\prime}(\omega)\right)$ and the sample length $(L)$ through the phase factor $\beta$ as

$$
\phi=-\beta L=-\frac{2 \pi \sqrt{\varepsilon^{\prime}(\omega)}}{\lambda_{0}} L
$$

where $\lambda_{0}$ is the wavelength in vacuum. The real part of the permittivity is then calculated from eq 17. After determination of $\varepsilon^{\prime}(\omega)$, the imaginary part of the complex permittivity $\varepsilon^{\prime \prime}(\omega)$ is iteratively obtained from the magnitude of $S_{21}$. In this predictor-corrector numerical procedure, an initial value for $\varepsilon^{\prime \prime}(\omega)$ is estimated, and the $S_{21}$ magnitude is calculated. The estimated $\varepsilon^{\prime \prime}(\omega)$ is then changed to minimize the difference between calculated and measured $S_{21}$. The data processing is carried out with a self-built computer program.

The uncertainty of the two-port transmission line technique can be divided into two parts. The first regards the accuracy of the measured $S$-parameter, which is primarily dependent on the $S_{21}$ magnitude and equipment precision. ${ }^{36}$ For our instrumentation and for a typical $S_{21}$ magnitude less than $-30 \mathrm{~dB}$, the error is better than $0.5 \%$, but it increases rapidly for $S_{21}$ magnitudes below -40 $\mathrm{dB}$. For example, for $S_{21}$ values below $-50 \mathrm{~dB}$, the measured error is in excess of $5 \%$. The second part is the accuracy of the calculated complex permittivity. To calculate the uncertainties in the permittivity $\left(\Delta \varepsilon^{\prime} / \varepsilon^{\prime}\right)$ and the dielectric loss $\left(\Delta \varepsilon^{\prime \prime} / \varepsilon^{\prime \prime}\right)$, it is necessary to calculate the partial derivative of the permittivity with respect to the $S$-parameter, $\left(\partial \varepsilon^{*}\right) /\left(\partial S_{21}\right)$. For static permittivity less than 100 , which is the case of polymer electrolytes, typical errors for the complex permittivity are $2 \%$ for $\varepsilon^{\prime}$ and $3 \%$ for $\varepsilon^{\prime \prime}$. Other contributions to the error arise from sample length measurements, air gaps around the sample, and waveguide mismatch. These error terms can be minimized by careful sample preparation and operation. Detailed uncertainty analysis of this technique can be found in ref 35 .

\section{Technique Validation and Experimental Section}

3.1. Validation of the Transmission Line Technique. A vector network analyzer HP8510C, with a broadband sweep generator synthesized signals from $45 \mathrm{MHz}$ to $26 \mathrm{GHz}$, was used to measure the complex transmission parameter $S_{21}$. A 7 $\mathrm{mm}$ coaxial line (10 cm long) was connected to HP8510C through two standard transmission lines with impedance of 50 $\Omega$. Before measurement, the system was calibrated with an open, a short, and a known load (50 $\Omega$ ). Calibration of $S_{21}$ magnitude was made by performing a simple through connection with air as the dielectric medium.

As a first evaluation of the two-port transmission line technique, the dielectric spectra of deionized water (Millipore, $18.2 \mathrm{M} \Omega \mathrm{cm}$ ) were measured at $25{ }^{\circ} \mathrm{C}$. Because of the high dielectric loss of water, several sample lengths, varying from 10 to $1 \mathrm{~cm}$, were used to obtain the optimal measurement for the various frequencies across the dielectric spectrum. The selection of the sample length was guided by the following empirical equation, which was obtained by Stuchly and Matuszewski ${ }^{37}$ and gave minimal experimental uncertainty:

$$
\frac{L}{\lambda}=\frac{1}{4}\left|\varepsilon^{*}(\omega)\right|^{-1 / 2}
$$
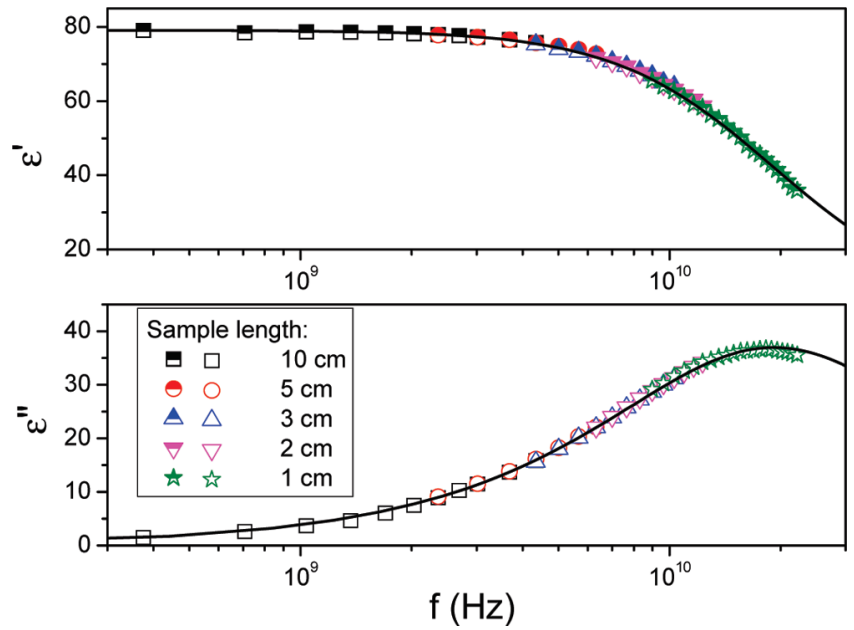

Figure 2. Complex permittivity spectra of liquid water in the frequency range of $10^{8} \mathrm{~Hz}$ to $26 \mathrm{GHz}$ obtained by varying sample lengths. The solid lines are the best fitting using one Debye relaxation process.

where $L / \lambda$ represents the normalized sample length. In the measurements of liquid water, the coaxial line was fixed in a vertical position, and $L_{1}$ was set to 0 . A thin layer of vacuum grease, whose dielectric properties are close to that of air, was placed in the probe connector to prevent liquid leakage. Similar approaches were also described in literature, where a dielectric plug was normally used to seal the liquid. ${ }^{38,39}$ The influence of the dielectric plug on the measurement was generally assumed to be negligible and was not taken into account in the theoretical formulation of the problem.

The dielectric spectra of water at $25^{\circ} \mathrm{C}$ are shown in Figure 2 . From this figure, $\varepsilon^{\prime}(\omega)$ falls with increasing frequency, and $\varepsilon^{\prime \prime}(\omega)$ shows a peak at around $18 \mathrm{GHz}$. The spectra are best fitted by a Debye relaxation model $(\alpha=0$ and $\beta=1$, see eq 20 ). The static permittivity from the best fitting is 79.13 , which agrees within $1 \%$ with literature data. ${ }^{40,41}$ The corresponding relaxation time is obtained to be $8.31 \mathrm{ps,} \mathrm{which} \mathrm{agrees} \mathrm{within}$ $0.5 \%$ with literature data. ${ }^{40,42}$

To assess the validity of the transmission line technique for the medium in film form, initial measurements were also made on thin strips of polytertrafluoroethylene (PTFE) film (McMaster, 0.003" thick, electronic grade) at different sample lengths. The dielectric spectra are shown in Figure 3 for two lengths, 5 and $1 \mathrm{~cm}$. In contrast to liquid water, the PTFE sample length has little, if any at all, influence on the dielectric spectra due to its low dielectric loss. The relative permittivity is constant at around 2.0 over the frequency range of $0.045-26 \mathrm{GHz}$. This result agrees well with the dielectric constant of 2.055 reported by Geyer and Krupka for Teflon at $9.93 \mathrm{GHz} .{ }^{43}$ The dielectric loss of PTFE film is found to be very small (in the order of 0.001 ) but exhibits scattering over the frequency range. The initial experiments on water and PTFE film indicate that the two-port transmission line technique is appropriate for the dielectric measurements of both aqueous and polymeric electrolytes.

3.2. Dielectric Spectra Measurements of Flemion SH150. In our previous work, we measured the dielectric spectra of hydrated Nafion 117 with the same two-port transmission line method and identified at least two water relaxations in the frequency range of $0.1-26 \mathrm{GHz} .{ }^{11}$ To study the dielectric relaxation behavior of the membranes having different equivalent weight $(\mathrm{EW})$, we measure in this work the microwave dielectric spectra of another perfluorated sulfonic acid membrane, Flemion SH150, which has a lower equivalent weight 


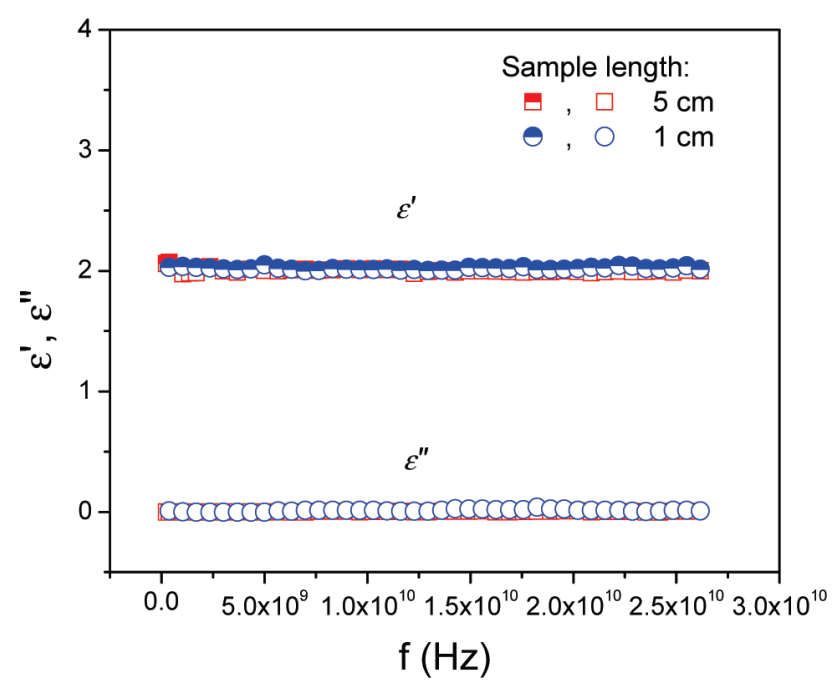

Figure 3. Complex permittivity spectra of Teflon in the frequency range of $10^{8} \mathrm{~Hz}$ to $20 \mathrm{GHz}$ obtained at two sample lengths of 5 and 1 $\mathrm{cm}$. The slight difference between two sample lengths is likely due to the error in determination of the sample length.

TABLE 1: Chemical Structure, Equivalent Weight (EW), and Thickness for Nafion 117 and Flemion SH 150

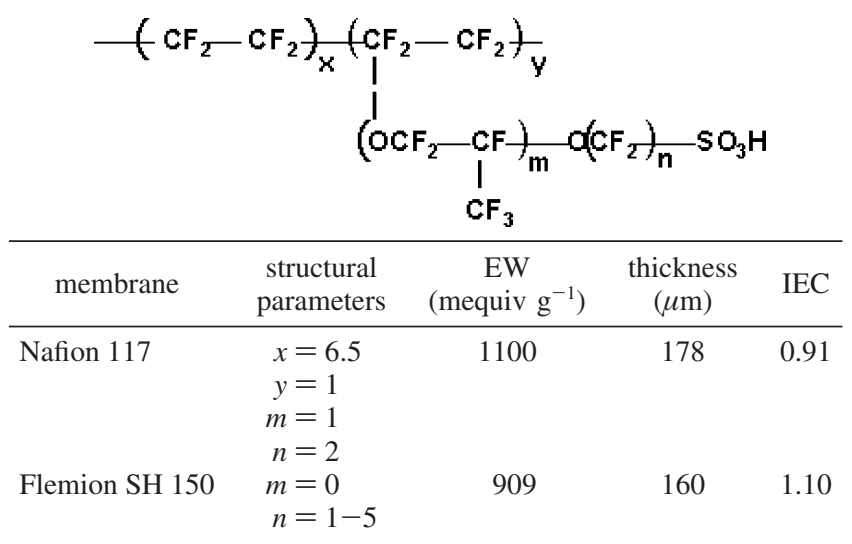

$(\mathrm{EW}=909 \mathrm{~g}$ /equiv versus $\mathrm{EW}=1100$ for Nafion 117). Table 1 lists the chemical structure and physical properties of Flemion SH150 and the corresponding values of Nafion 117. It is expected that EW is an important factor in determining the phase-separated structure of the hydrated membrane and thus the dynamics of protons and water molecules within the membrane.

The Flemion membranes were treated by boiling in $3 \% \mathrm{H}_{2} \mathrm{O}_{2}$, rinsing in boiling water, then boiling in $0.5 \mathrm{M} \mathrm{H}_{2} \mathrm{SO}_{4}$, and finally rinsing in boiling water. The membranes were then hydrated in closed jars where the relative humidity was controlled by different salt solutions at $25{ }^{\circ} \mathrm{C}$. Three RHs, 33\%, 75\% and $100 \%$, were used, and the corresponding water contents $\lambda$ were $4.5,7.1$, and 13.5 , respectively ( $\lambda$, denoting the number of absorbed water molecules per $-\mathrm{SO}_{3} \mathrm{H}$ site). The "dry" membrane was obtained by drying under vacuum at $120^{\circ} \mathrm{C}$ for $12 \mathrm{~h}$. The water content for the dry membrane is estimated as $\lambda \approx 1$. The pretreated Flemion membranes were carefully wrapped around the inner conductor of the coaxial line. After the installation of the sample, the coaxial line (containing the sample) was conditioned again in the same salt solution to restore the membrane water content. The coaxial line was then quickly connected to the network analyzer HP8510C, and the transmission $S$-parameter $\left(S_{21}\right)$ was collected over the entire frequency range. The measurement usually lasted less than 5 min, and no variation in membrane water content was found. Two sample lengths, 3 and $1 \mathrm{~cm}$, are used to obtain the optimal dielectric spectra over the entire frequency range at $25^{\circ} \mathrm{C}$.

\section{Results and Discussion}

4.1. Data Analysis. For each measurement, the total loss of the sample, $\eta^{\prime \prime}(\omega)$, together with the dielectric permittivity, $\varepsilon^{\prime}(\omega)$, was numerically calculated from the measured $S_{21}$ spectrum. According to eq 1 , the true dielectric loss of the electrolytes, $\varepsilon^{\prime \prime}(\omega)$, can only be obtained from $\eta^{\prime \prime}(\omega)$ after the correction for the conductivity contribution:

$$
\eta_{\mathrm{k}}^{\prime \prime}=\frac{\sigma}{\omega \varepsilon_{0}}
$$

The dc conductivity, $\sigma$, is treated as an adjustable parameter in the data analysis.

After subtraction of the conductivity contribution, the reduced dielectric spectra, $\varepsilon^{*}(\omega)=\varepsilon^{\prime}(\omega)-\mathrm{j} \varepsilon^{\prime \prime}(\omega)$, are fitted to a superposition of multiple Havriliak-Negami equations ${ }^{11}$ or variants thereof. ${ }^{44,45}$

$$
\begin{array}{r}
\varepsilon^{*}(\omega)=\varepsilon_{\infty}+\sum_{i=1}^{n} \frac{\Delta \varepsilon_{i}}{\left[1+\left(j \omega \tau_{i}\right)^{\left.1-\alpha_{i}\right]_{i}}\right.} \text { with } \Delta \varepsilon_{i}=\varepsilon_{s i}- \\
\varepsilon_{\infty i}, 0 \leq \alpha_{i} \leq 1,0 \leq \beta_{i} \leq 1
\end{array}
$$

where $n$ is the number of separable dispersion steps, $\varepsilon_{s i}$ and $\varepsilon_{\infty i}$ are the low-high-frequency permittivity limits for the $i$ th process from the lower frequency side, respectively, and $\omega=2 \pi f$ is the angular frequency, $\tau$ is the dielectric relaxation time, and $\alpha$ and $\beta$ are shape parameters for the distribution of relaxation times. This general equation can be reduced to three well-known models as limiting cases: the Debye equation $(\alpha=0, \beta=$ $1)$, the Cole-Cole equation $(0 \leq \alpha<1, \beta=1)$, and the Davidson-Cole equation ( $\alpha=0,0<\beta \leq 1)$. The relative permittivity spectra, $\varepsilon^{*}(\omega)$, are analyzed by simultaneously fitting $\varepsilon^{\prime}(\omega)$ and $\varepsilon^{\prime \prime}(\omega)$ with a least-squares procedure, based on the Levenberg-Marquardt algorithm (for more details, see also ref 11$)$. The program allows one to model $\varepsilon^{*}(\omega)$ as the sum of multiple dispersion steps, where for each relaxation process a band shape defined by Debye, Cole-Cole, Davidson-Cole, or Havriliak-Negami equation can be selected. For most of the samples, the best results (that is, the minimum variance, $\chi^{2}$, of the fit and a consistent set of relaxation parameters as a function of water content) were obtained with a sum of two Debye relaxation processes $(\alpha=0, \beta=1)$.

4.2. Microwave Dielectric Spectra of Flemion SH150. Figure 4 shows the dielectric spectra of the "dry" Flemion membrane along with the results of the "dry" Nafion $117 .{ }^{11}$ No relaxation process can be identified from the absorption spectra in the studied frequency range. Dry Flemion SH150 and dry Nafion 117 have very similar values of permittivity and dielectric loss, which indicates that the chemical structure and the equivalent weight have little effect on the dielectric properties of dry PFSA membranes. The higher values of $\varepsilon^{\prime}$ and $\varepsilon^{\prime \prime}$ of both dry PFSA polymers as compared to those of PTFE (see Figure 3) are due to the presence of sulfonic acid groups in these membranes (whereas PTFE is largely apolar).

The dielectric spectra of the hydrated Flemion membranes are remarkably different from those of the dry samples. Figure 5 shows the total loss $\eta^{\prime \prime}(\omega)$ of Flemion samples with water contents of $\lambda=4.5,7.1$, and 13.5, equilibrated at RH of $33 \%$, 


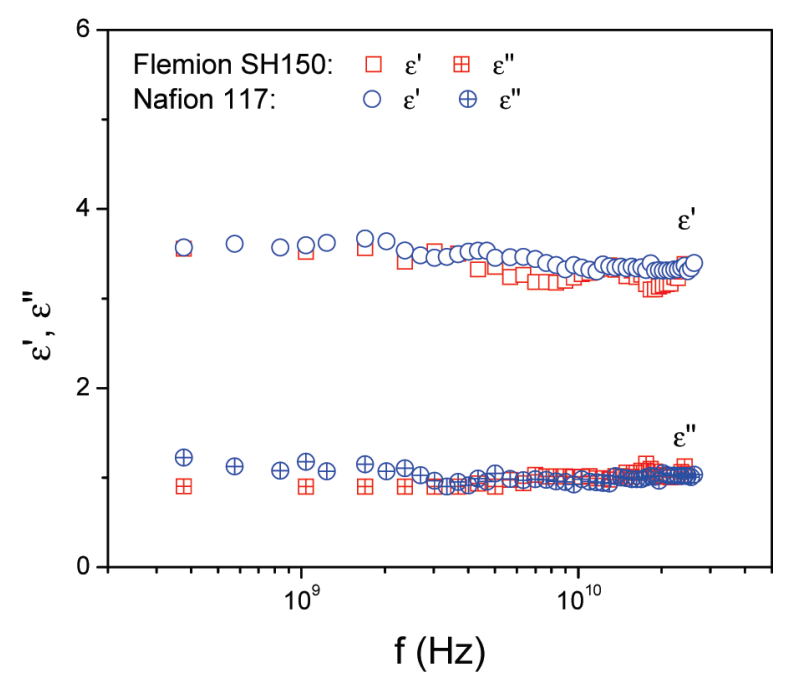

Figure 4. Relative permittivity, $\varepsilon^{\prime}(\omega)$, and dielectric loss spectrum, $\varepsilon^{\prime \prime}(\omega)$, of dry Flemion SH150 at $25{ }^{\circ} \mathrm{C}$. The dielectric spectra for dry Nafion 117 are also included.

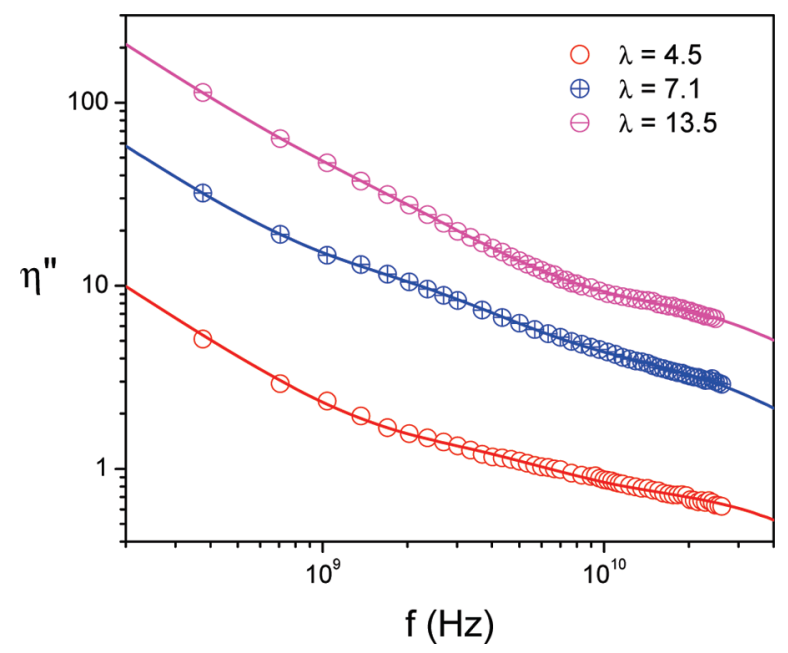

Figure 5. Total loss $\eta^{\prime \prime}(\omega)$ of Flemion samples with water contents of $\lambda=4.5,7.1$, and 13.5 , pretreated at RH of $33 \%, 75 \%$, and $100 \%$, respectively. The linear portion of the fitted lines at low frequencies shows the conductivity contribution. The detailed fittings at higher frequencies are shown in the following figures.

$75 \%$, and $100 \%$, respectively. The contribution of the conductivity due to the long-range motion of $\mathrm{H}^{+} / \mathrm{H}_{2} \mathrm{O}$ is clearly shown in the low-frequency range and follows an inverse frequency $\left(f^{-1}\right)$ dependence. The conductivity values of the hydrated samples are obtained from data fitting and are listed in Table 2. Flemion SH150 membranes show comparable conductivities to Nafion 117 membranes for similar water contents. It is difficult to compare the fitted Flemion conductivities to literature data, due to the scarcity of the conductivity data of Flemion membranes.

After the contribution of the conductivity is subtracted from the total loss according to eq 2 , the true dielectric loss $\varepsilon^{\prime \prime}(\omega)$ is obtained. At least two dielectric relaxations are clearly seen from the $\varepsilon^{\prime \prime}(\omega)$ raw spectra. The dielectric spectra are then analyzed by the various relaxation models of eq 20. The best common fit of the spectra $\varepsilon^{\prime}(\omega)$ and $\varepsilon^{\prime \prime}(\omega)$ is obtained by a superposition of two Debye relaxations. Figure 6 shows the dielectric response of the hydrated Flemion membranes at $25^{\circ} \mathrm{C}$ with $\lambda=4.5$ and 7.1 , along with the fitted curves corresponding to two Debye relaxations. The two relaxation processes are essentially identical to the two relaxation modes observed in hydrated Nafion $117 .{ }^{11}$
The higher-frequency process coincides with the dynamics of pure water (see Figure 2 and also ref 40), with the maximum frequency at about $18 \mathrm{GHz}$, and is therefore attributed to the dielectric relaxation of the bulk-like water regions within the membrane. This dielectric relaxation corresponds to the ordinary water behavior dominated by water-water hydrogen bonding, ${ }^{40}$ and we attribute it to water domains located in regions where the local environment resembles bulk/liquid water. The lowerfrequency process in Figure 6, having a longer relaxation time ranging from 20 to $70 \mathrm{ps}$, is attributed to water molecules loosely bound to the PFSA. This water relaxation is almost identical in nature as observed before ${ }^{11}$ for Nafion 117 , and the conclusions follow along the same lines as before. ${ }^{11}$ This lower-frequency process clearly corresponds to water molecules that are neither unconstrained/bulk-like water, nor directly coordinated to sulfonates. The water molecules that are tightly bound to the sulfonic sites (also called hydration water) display a relaxation in the $\mathrm{MHz}$ range $\mathrm{e}^{11}$ (with a corresponding relaxation time of about $10^{-7} \mathrm{~s}$ ) and thus cannot be captured in the microwave range. Because of the broad distribution of environments within the PFSA membrane, the loosely bound water can exist in many different local environments: ${ }^{11}$ (i) water in second hydration shell surrounding the strongly bound water to the sulfonates, (ii) water in hydrophilic channels connecting the hydrated ionic clusters, or (iii) water at the interface between the polymer and the bulklike liquid water as well as water confined in the hydrophobic domains of the membrane (i.e., hydrophobic hydration water). These environments are inherently heterogeneous in dynamics and geometries, ${ }^{46-53}$ and although a single Debye relaxation fits well the loosely bound water dynamics, the data do not necessarily denote that the loosely bound water dynamics display a single-relaxation-time (monoexponential) mode; rather, the single Debye relaxation reflects a well-equilibrated hydrated structure of the polymer (more detailed discussion on this can be found in ref 11). The dielectric parameters for the hydrated Flemion membranes are summarized in Table 2.

The relative permittivity $\left(\varepsilon^{\prime}(\omega)\right)$ and absorption $\left(\varepsilon^{\prime \prime}(\omega)\right)$ spectra for Flemion SH150 with $\lambda=13.5$, equilibrated at $100 \%$ $\mathrm{RH}$, are shown in Figure 7. The spectra for $\lambda=13.5 \mathrm{did}$ not yield satisfactory fittings of the lower-frequency peak despite the fact that the bulk-like water mode (higher-frequency peak) is fitted with good certainty. The same situation has also been found for Nafion 117 equilibrated at 100\% RH (ref 11, Appendix). The noise of the permittivity data at the lower frequencies in the case of vapor saturated PFSA samples may be due to the water condensation inside the coaxial line.

The dielectric strengths (or dielectric dispersions, $\Delta \varepsilon$ ), which can be considered as a measure of the population of water molecules that contribute to a specific relaxation, for the two $\mathrm{GHz}$ water modes in Flemion $\mathrm{SH} 150$ at $25^{\circ} \mathrm{C}$, as derived from the data analysis, are shown in Figure 8. For comparison, the corresponding values for Nafion 117 are also included in this figure. The bars for the loosely bound water at $\lambda=13.5$ for Flemion and $\lambda=12$ for Nafion samples are due to data scattering at low frequencies and indicate ranges of $\Delta \varepsilon$ that can yield equally good fits to the raw data/spectra. Similar behavior is found for Flemion SH150 and Nafion 117 with respect to the variation of the dielectric strengths as a function of water content. The loosely bound water initially increases with increased hydration and subsequently levels off at higher $\lambda$, while the bulk-like water is low at low water content and then increases almost linearly with $\lambda$. It can be reasonably assumed that the bulk-like water will become dominant at the higher hydration levels. It is also clear from Figure 8 that Flemion 
TABLE 2: Dielectric Parameters Best Fitted by a Sum of Two Debye Relaxation Processes for Flemion SH150 Membranes with Three Water Contents ${ }^{a}$

\begin{tabular}{|c|c|c|c|c|c|c|}
\hline system & $\Delta \varepsilon_{\text {l.f. }}$ & $\tau_{\text {l.f. }}(\mathrm{ps})$ & $\Delta \varepsilon_{\text {h.f. }}$ & $\tau_{\text {h.f. }}(\mathrm{ps})$ & $\varepsilon_{\infty}$ & $\sigma_{\mathrm{fit}}, \mathrm{S} / \mathrm{m}$ \\
\hline $\mathrm{H}_{2} \mathrm{O}$ & & & $73.9 \pm 0.08$ & $8.30 \pm 0.01$ & 5.2 (fixed) & \\
\hline $\begin{array}{l}\text { Flemion } \mathrm{SH} 150-\mathrm{H}_{2} \mathrm{O} \\
\lambda=4.5(\mathrm{RH} 33 \%) \\
\lambda=7.1(\mathrm{RH} 75 \%) \\
\lambda=13.5(\mathrm{RH} 100 \%)\end{array}$ & $\begin{array}{c}1.3 \pm 0.1 \\
8.9 \pm 0.2 \\
(11.3-14.8)\end{array}$ & $\begin{array}{l}39.48 \pm 1.50 \\
69.58 \pm 1.70\end{array}$ & $\begin{array}{l}0.6 \pm 0.1 \\
3.6 \pm 0.1 \\
9.5 \pm 0.4\end{array}$ & $\begin{array}{l}8.08 \pm 0.30 \\
8.19 \pm 0.35 \\
8.03 \pm 0.30\end{array}$ & $\begin{array}{l}3.0 \pm 0.1 \\
3.9 \pm 0.1 \\
3.8 \pm 0.1\end{array}$ & $\begin{array}{l}0.11 \pm 0.02 \\
0.63 \pm 0.04 \\
2.29 \pm 0.07\end{array}$ \\
\hline $\begin{array}{l}\text { Nafion }-\mathrm{H}_{2} \mathrm{O}^{11} \\
\lambda=3.0(\mathrm{RH} 33 \%) \\
\lambda=6.0(\mathrm{RH} 75 \%) \\
\lambda=9.0(\text { RH } 96 \%) \\
\lambda=12.0(\text { RH } 100 \%)\end{array}$ & $\begin{array}{c}1.1 \pm 0.1 \\
1.4 \pm 0.1 \\
5.1 \pm 0.2 \\
(4.3-5.9)\end{array}$ & $\begin{array}{l}24.40 \pm 1.45 \\
25.63 \pm 1.30 \\
37.08 \pm 1.20\end{array}$ & $\begin{array}{r}(0.0-0.5) \\
0.6 \pm 0.1 \\
3.5 \pm 0.1 \\
6.8 \pm 0.5\end{array}$ & $\begin{array}{l}8.72 \pm 0.80 \\
8.96 \pm 0.75 \\
8.76 \pm 0.45 \\
8.27 \pm 0.30\end{array}$ & $\begin{array}{l}3.0 \pm 0.1 \\
3.2 \pm 0.1 \\
3.9 \pm 0.1 \\
3.7 \pm 0.1\end{array}$ & $\begin{array}{l}0.22 \pm 0.02 \\
0.34 \pm 0.02 \\
1.78 \pm 0.03 \\
2.05 \pm 0.04\end{array}$ \\
\hline
\end{tabular}

${ }^{a}$ The results for liquid water and hydrated Nafion 117 membranes ${ }^{11}$ are also included for comparison. $\Delta \varepsilon$ represents dielectric strength, $\tau$ relaxation time, $\varepsilon_{\infty}$ high-frequency permittivity limit, and $\sigma$ conductivity. The subscripts 1.f. and h.f. represent lower-frequency and higher-frequency relaxation processes, respectively.
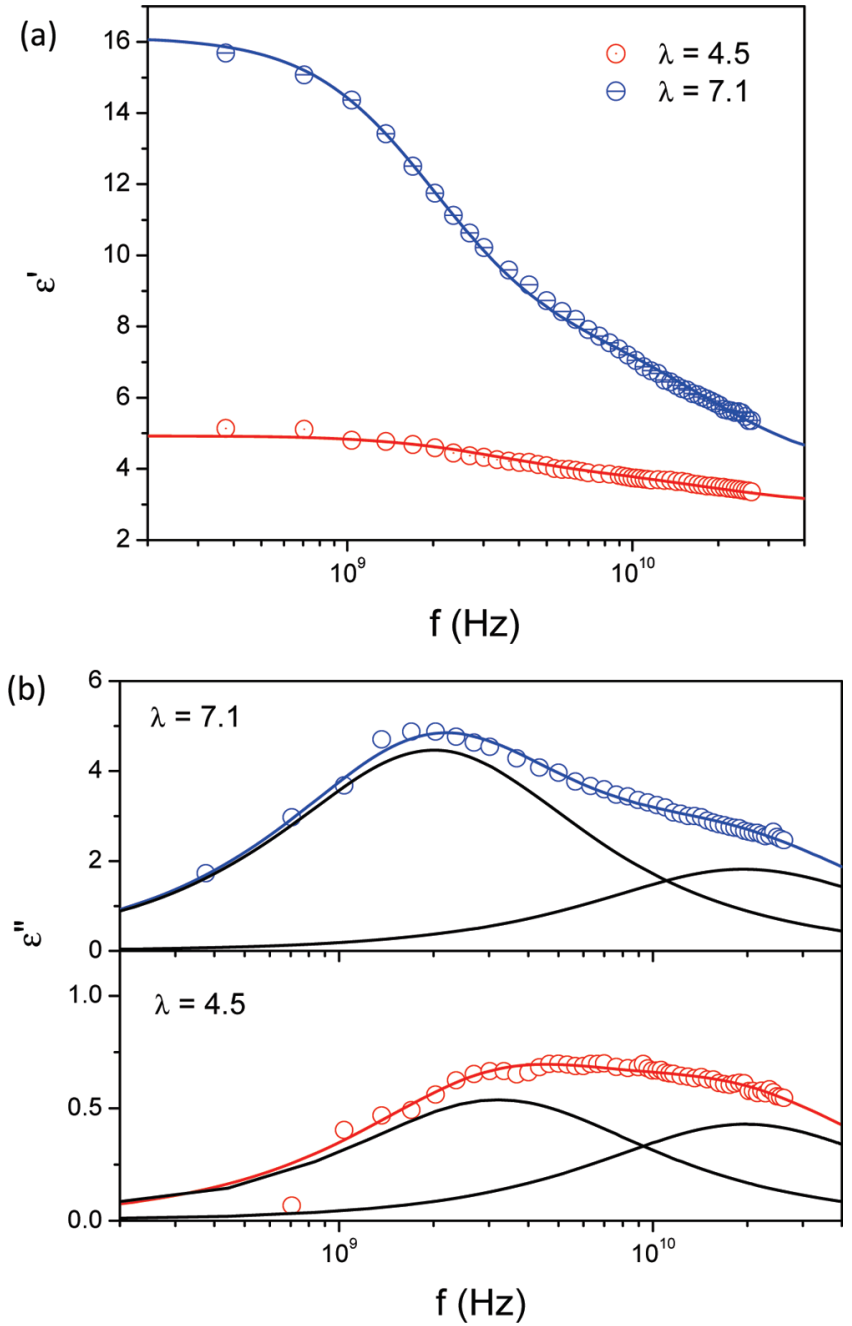

Figure 6. Permittivity spectra versus frequency at $25^{\circ} \mathrm{C}$ for Flemion SH150 with water content of $\lambda=4.5$ and 7.1: (a) relative permittivity $\varepsilon^{\prime}(\omega)$ and (b) dielectric loss $\varepsilon^{\prime \prime}(\omega)$. The solid lines represent the corresponding best fits by two Debye processes. The faster process (higher-frequency peak) corresponds to bulk-like water and the slower process (lower-frequency peak) to loosely bound water.

shows higher dielectric strengths than Nafion for both the loosely bound water and the bulk-like water, which is a naturally expected consequence of the difference in polymer structure (cf., the difference in EW between the two polymers). The dielectric responses of the PFSA membranes mainly result from

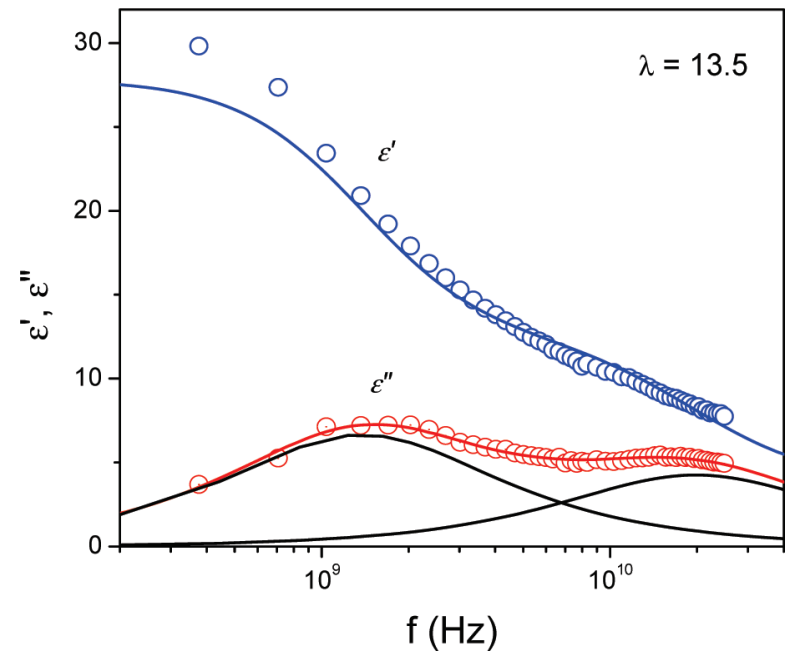

Figure 7. Relative permittivity $\left(\varepsilon^{\prime}(\omega)\right)$ and absorption $\left(\varepsilon^{\prime \prime}(\omega)\right)$ spectra for Flemion SH150 with $\lambda=13.5$ at $25{ }^{\circ} \mathrm{C}$; this membrane was equilibrated in saturated water vapor (relative humidity $=100 \%$ ). The solid lines are the fits by two Debye processes.

the hydrated ionic cluster regions in the membrane, as demonstrated on the basis of the comparison among Figures 4, 6, and 7 as well as from the literature. $5,28,29,54$ The structural parameters that should have important effects on the dielectric properties of the membrane include the size and/or number of the ionic cluster regions, which are dependent on the membrane water content ${ }^{55,56}$ and the equivalent weight. ${ }^{13}$ The higher dielectric strengths of Flemion SH150 (EW = 909) are in agreement with the expectation that at lower EW larger size and/or larger number of hydrated ionic clusters can be formed (as compared to a membrane with higher EW, i.e., Nafion with $\mathrm{EW}=1100$, at the same hydration level). However, because Flemion SH150 and Nafion 117 are synthesized by different manufacturers, no further analysis is possible on quantifying the effect of equivalent weight without precise knowledge of the two polymers' microstructure.

\section{Conclusions}

In this work, a two-port transmission technique for measuring the complex permittivity spectra of polymeric electrolytes in the microwave region is described. An overview of the theoretical background of the transmission line equations and an iterative approach for solving the scattering equations are also presented. The ability of this technique to accurately 


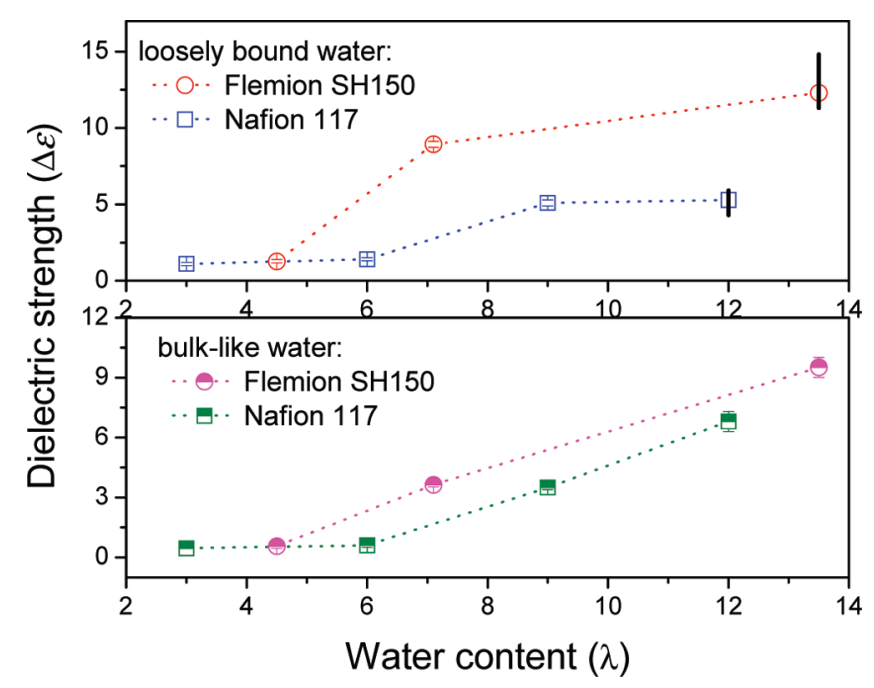

Figure 8. Dielectric strengths and the corresponding uncertainties for the two water modes (bulk-like and loosely bound water) observed at microwave frequencies in the hydrated Flemion SH150 samples at 25 ${ }^{\circ} \mathrm{C}$. For comparison, the corresponding values for Nafion $117^{11}$ are also included. The bars for the loosely bound water at $\lambda=13.5$ for Flemion and $\lambda=12$ for Nafion samples are due to data scattering at low frequencies.

measure permittivity spectra is experimentally validated with liquid water and polytertrafluoroethylene films. In the case of liquid water, multiple sample lengths are required to obtain the optimal measurement of the dielectric spectra across the whole experimental frequency range due to the high dielectric losses, while for PTFE a single sample length is sufficient to cover the entire frequency range.

The dielectric relaxation spectra of dry and hydrated Flemion SH150 membranes, which has a chemical structure similar to that of Nafion 117, but has a lower equivalent weight $(\mathrm{EW}=$ 909 g/equiv vs Nafion's EW $=1100$ g/equiv), are then measured with the same two-port transmission line technique over the frequency range from $45 \mathrm{MHz}$ to $26 \mathrm{GHz}$ at $25{ }^{\circ} \mathrm{C}$. The permittivity spectra are well-fitted by a superposition of two Debye relaxation peaks. These relaxation processes are essentially identical to those observed in a hydrated Nafion 117 membrane. The higher-frequency peak, with a relaxation time of about 8 ps at $25^{\circ} \mathrm{C}$, shows the characteristic dynamics of liquid water and is attributed to the cooperative relaxation of free/bulk-like water. The lower-frequency peak, characterized by longer relaxation times of $20-70 \mathrm{ps}$, is attributed to water molecules that are loosely bound to the membrane. The amount of the bulk-like water, quantified through the dielectric strength of each corresponding relaxation mode, increases linearly with increasing water content, while the loosely bound water increases with increased hydration and levels off at higher water contents. Flemion SH150 displays higher dielectric strengths for both these water modes and therefore higher dielectric constant than Nafion 117, which reflects the effect of the equivalent weight on the polymer structure, particularly on the phase-separated hydrated ionic cluster domains.

Acknowledgment. Z.L. acknowledges Mr. Steve Perini for his help in the experimentation and Mr. Khalid Rajab for his assistance in software. We gratefully acknowledge financial support for this work by International Fuel Cells Inc. through subcontract no. 3540OB via Contract No. DE-FCo4-02AL67608 and by the U.S. Department of Energy via Contract No. DEFG02-07ER46371 at Penn State University. E.M. additionally acknowledges support from the National Science Foundation (NSF grant \# DMR-0602877).

\section{References and Notes}

(1) Kreuer, K. D. In Handbook of Fuel Cells Fundamentals, Technology and Applications; Vielstich, W., Lamm, A., Gasteiger, H. A., Eds.; John Wiley \& Sons: Chichester, UK, 2003; Vol. 3, Chapter 33.

(2) Mortimer, R. J.; Beech, A. Electrochim. Acta 2002, 47, 3383.

(3) Michishita, H.; Matsumoto, H.; Ishihara, T. Electrochemistry 2008, 76, 288.

(4) Hsu, W. Y.; Gierke, T. D. J. Membr. Sci. 1983, 13, 307.

(5) Kreuer, K. D. J. Membr. Sci. 2001, 185, 29.

(6) Mauritz, K. A.; Moore, R. B. Chem. Rev. 2004, 104, 4535.

(7) Doyle, M.; Rajendran, G. In Handbook of Fuel Cells Fundamentals, Technology and Applications; Vielstich, W., Lamm, A., Gasteiger, H. A., Eds.; John Wiley \& Sons: Chichester, UK, 2003; Vol. 3, Chapter 30.

(8) Paul, R.; Paddison, S. J. J. Phys. Chem. B 2004, 108, 13231.

(9) Paul, R.; Paddison, S. J. J. Chem. Phys. 2005, 123, 224704.

(10) Paddison, S. J.; Paul, R.; Zawodzinski, T. A., Jr. J. Electrochem. Soc. 2000, 147, 617 .

(11) Lu, Z.; Polizos, G.; Macdonald, D. D.; Manias, E. J. Electrochem. Soc. 2008, 155, B163.

(12) Paddison, S. J. Annu. Rev. Mater. Res. 2003, 33, 289.

(13) Saito, M.; Arimura, N.; Hayamizu, K.; Okada, T. J. Phys. Chem. B 2004, 108, 16064 .

(14) Paddison, S. J.; Paul, R. Phys. Chem. Chem. Phys. 2002, 4, 1158.

(15) Kreuer, K. D.; Paddison, S. J.; Spohr, E.; Schuster, M. Chem. Rev.

2004, 104, 4637.

(16) Kornyshev, A. A.; Kuznetsov, A. M.; Spohr, E.; Ulstrup, J. J. Phys. Chem. B 2003, 107, 3351 .

(17) Yeo, S. C.; Eisenberg, A. J. Appl. Polym. Sci. 1977, 21, 875.

(18) Tsonos, C.; Apekis, L.; Pissis, P. J. Mater. Sci. 2000, 35, 5957.

(19) Tsonos, C.; Apekis, L.; Pissis, P. J. Mater. Sci. 1998, 33, 2221.

(20) Di Noto, V.; Lavina, S.; Negro, E.; Vittadello, M.; Conti, F.; Piga,

M.; Pace, G. J. Power Sources 2009, 187, 57.

(21) Mauritz, K. A. Macromolecules 1989, 22, 4483.

(22) Deng, Z. D.; Mauritz, K. A. Macromolecules 1992, 25, 2739.

(23) Starkweather, H. W.; Chang, J. J. Macromolecules 1982, 15, 752.

(24) Baker-Jarvis, J.; Janezic, M. D.; Geyer, R. G. IEEE Trans. Instrum. Meas. 1994, 43, 711 .

(25) Wei, Y.; Sridhar, S. IEEE Trans. Microwave Theory Tech. 1991, $39,526$.

(26) Wei, Y.; Sridhar, S. J. Chem. Phys. 1990, 92, 923.

(27) Misra, D. K. Microwave Opt. Technol. Lett. 1996, 11, 183.

(28) Paddison, S. J.; Reagor, D. W.; Zawodzinski, T. A., Jr. J. Electroanal. Chem. 1998, 459, 91.

(29) Paddison, S. J.; Bender, G.; Kreuer, K. D.; Nicoloso, N.; Zawodzinski, T. A. J. New Mater. Electrochem. Syst. 2000, 3, 291.

(30) Jenkins, S.; Hodgetts, T. E.; Clarke, R. N.; Preece, A. W. Meas. Sci. Technol. 1990, 1, 691 .

(31) Baker-Jarvis, J.; Vanzura, E. J.; Kissick, W. A. IEEE Trans. Microwave Theory Techn. 1990, 38, 1096.

(32) Nicolson, A. M.; Ross, G. F. IEEE Trans. Instrum. Meas. 1968, IM-17-395.

(33) Kerns, D. M.; Beatty, R. W. Basic Theory of Waveguide Junctions and Introductory Microwave Network Analysis; Pergamon Press: New York, 1967.

(34) Weir, W. B. Proc. IEEE 1970, 62, 33.

(35) Lanagan, M. T. Ph.D. Dissertation, The Pennsylvania State University, 1987.

(36) Hewlett-Packard, Technical Data, 16, 1984.

(37) Stuchly, S. S.; Matuszewski, M. IEEE Trans. Instrum. Meas. 1978, IM-27-285.

(38) Somlo, P. I. IEEE Trans. Instrum. Meas. 1993, 42, 213.

(39) Qaddoumi, N.; Ganchev, S.; Zoughi, R. Res. Nondestruct. Eval. 1996, 8,177 .

(40) Kaatze, U. J. Chem. Eng. Data 1989, 34, 371.

(41) Ellison, W. J.; Lamkaouchi, K.; Moreau, J. M. J. Mol. Liq. 1996, $68,171$.

(42) Barthel, J.; Bachhuber, K.; Buchner, R.; Hetzenauer, H. Chem. Phys. Lett. 1990, 165, 369.

(43) Geyer, R. G.; Krupka, J. IEEE Trans Instrum. Meas. 1995, 44, 329.

(44) Havriliak, S.; Negami, S. Polymer 1967, 8, 161.

(45) Kremer, F.; Schönhals, A. Broadband Dielectric Spectroscopy; Springer: New York, 2002.

(46) Manias, E.; Kuppa, V. Eur. Phys. J. E 2002, 8, 193.

(47) Kuppa, V.; Foley, T. M. D.; Manias, E. Eur. Phys. J. E 2003, 12, 159.

(48) Manias, E.; Kuppa, V.; Yang, D. K.; Zax, D. B. Colloids Surf., A 2001, 187, 509. 
(49) Manias, E.; Hadziioannou, G.; ten Brinke, G. J. Chem. Phys. 1994, 101, 1721 .

(50) Manias, E.; Subbotin, A.; Hadziioannou, G.; ten Brinke, G. Mol. Phys. 1995, 85, 1017.

(51) Manias, E.; Hadziioannou, G.; ten Brinke, G. Langmuir 1996, 12, 4587.

(52) Giannelis, E. P.; Krishnamoorti, R.; Manias, E. Adv. Polym. Sci. 1999, 138, 107.
(53) Zhang, J.; Manias, E.; Wilkie, C. A. J. Nanosci. Nanotechnol. 2008, $8,1597$.

(54) Paul, P. R.; Paddison, S. J. J. Chem. Phys. 2001, 115, 7762.

(55) Gierke, T. D.; Munn, G. E.; Wilson, F. C. J. Polymer Sci., Polym. Phys. 1981, 19, 1687.

(56) Gebel, G. Polymer 2000, 41, 5829.

JP9057115 